University of Wollongong

Research Online

Australian Institute for Innovative Materials -

Papers

Australian Institute for Innovative Materials

$1-1-2018$

\title{
Enhancing the critical current of YBa2Cu307 thin films by substrate nanoengineering
}

Antony Jones

University of Wollongong, anj093@uowmail.edu.au

Simon K. H Lam

CSIRO

Jia Du

CSIRO

Sergey Rubanov

University of Melbourne, sergey@unimelb.edu.au

Alexey Pan

University of Wollongong, pan@uow.edu.au

Follow this and additional works at: https://ro.uow.edu.au/aiimpapers

Part of the Engineering Commons, and the Physical Sciences and Mathematics Commons

Research Online is the open access institutional repository for the University of Wollongong. For further information contact the UOW Library: research-pubs@uow.edu.au 


\title{
Enhancing the critical current of $\mathrm{YBa} 2 \mathrm{Cu} 307$ thin films by substrate nanoengineering
}

\author{
Abstract \\ The critical current density, $\mathrm{J} \mathrm{c}$, can be increased by introducing defects throughout $\mathrm{YBa} 2 \mathrm{Cu} 307$ \\ superconducting thin films. We propose a new approach of substrate nanoengineering to produce well- \\ controlled defects. LaAlO 3 substrates have been ion-etched with different $34 \mathrm{~nm}$ deep patterns prior to \\ the deposition of the films. An annealing step at $1000^{\circ} \mathrm{C}$ after substrate etching has been undertaken to \\ negate the Ar-ion damage to the surface. The $\mathrm{J} \mathrm{c}$ of the so-prepared samples measured at different \\ temperatures has been compared to thin films deposited on plain substrates at the same time. In general, \\ an increase in $\mathrm{J} \mathrm{c}$ is observed across all temperatures and fields as a result of both patterning and \\ annealing. In particular, at zero field and $85 \mathrm{~K}$, the largest enhancement of $>40 \%$ has been recorded for \\ circle and triangle patterns. This new substrate nanoengineering technique is very promising for flux \\ trapping of superconducting devices, particularly because an increase to critical current (I c) also occurs. \\ Further optimization of depth, size, and shape of the patterns is expected to produce further \\ improvements to $\mathrm{J} \mathrm{c}$.

\section{Disciplines} \\ Engineering | Physical Sciences and Mathematics

\section{Publication Details} \\ Jones, A., Lam, S. K. H., Du, J., Rubanov, S. \& Pan, A. V. (2018). Enhancing the critical current of \\ YBa2Cu3O7 thin films by substrate nanoengineering. Journal of Applied Physics, 124 (23), \\ 233905-1-233905-6.
}




\section{Enhancing the critical current of $\mathrm{YBa}_{2} \mathrm{Cu}_{3} \mathrm{O}_{7}$ thin films by substrate nanoengineering}

Cite as: J. Appl. Phys. 124, 233905 (2018); https://doi.org/10.1063/1.5063261

Submitted: 25 September 2018 . Accepted: 01 December 2018 . Published Online: 20 December 2018

A. Jones (D, S. K. H. Lam, J. Du, S. Rubanov, and A. V. Pan
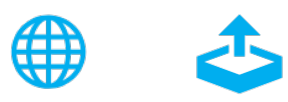

View Online

Export Citation

\section{ARTICLES YOU MAY BE INTERESTED IN}

Modified dispersion law for spin waves coupled to a superconductor

Journal of Applied Physics 124, 233903 (2018); https://doi.org/10.1063/1.5077086

Modifying the linear and nonlinear optical susceptibilities of coupled quantum dot-metallic nanosphere systems with the Purcell effect

Journal of Applied Physics 124, 233103 (2018); https://doi.org/10.1063/1.5063752

Magnified photonic spin-Hall effect with curved hyperbolic metamaterials

Journal of Applied Physics 124, 233104 (2018); https://doi.org/10.1063/1.5078634

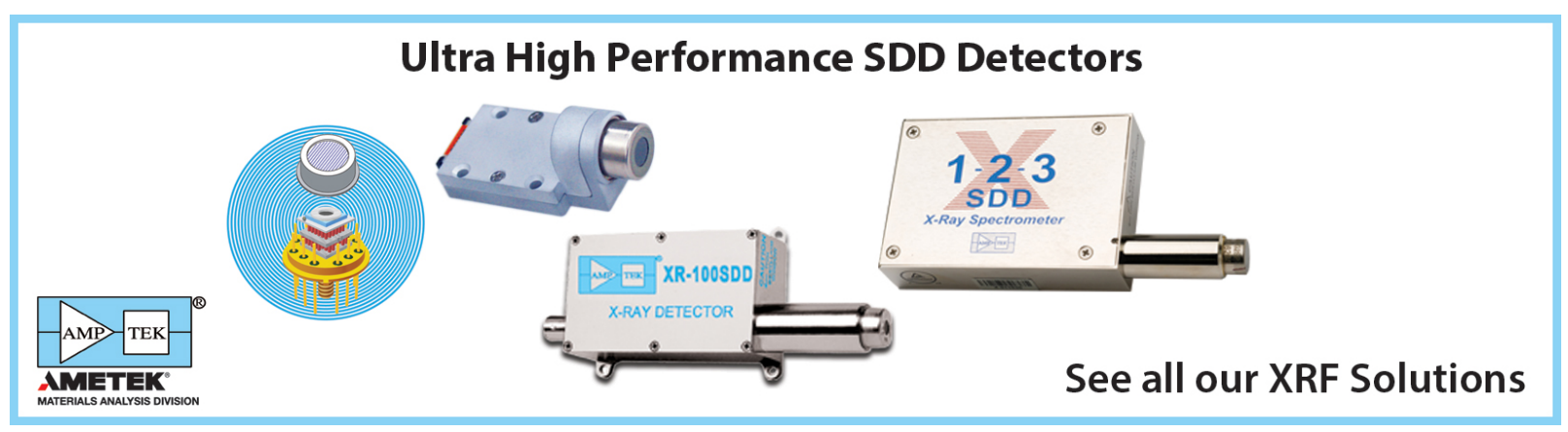




\title{
Enhancing the critical current of $\mathrm{YBa}_{2} \mathrm{Cu}_{3} \mathrm{O}_{7}$ thin films by substrate nanoengineering
}

\author{
A. Jones, ${ }^{1,2}$ S. K. H. Lam, ${ }^{2}$ J. Du, ${ }^{2}$ S. Rubanov, ${ }^{3}$ and A. V. Pan ${ }^{1,4, a)}$ \\ ${ }^{1}$ Institute for Superconducting and Electronic Materials, University of Wollongong, Northfields Avenue, \\ Wollongong, New South Wales 2522, Australia \\ ${ }^{2}$ CSIRO Manufacturing, Bradfield Road, West Lindfield, New South Wales 2070, Australia \\ ${ }^{3}$ Electron Microscope Unit, Bio21 Institute, University of Melbourne, Victoria 3010, Australia \\ ${ }^{4}$ National Research Nuclear University MEPhI (Moscow Engineering Physics Institute), 31 Kashirskoye \\ Shosse, 115409 Moscow, Russian Federation
}

(Received 25 September 2018; accepted 1 December 2018; published online 20 December 2018)

\begin{abstract}
The critical current density, $J_{c}$, can be increased by introducing defects throughout $\mathrm{YBa}_{2} \mathrm{Cu}_{3} \mathrm{O}_{7}$ superconducting thin films. We propose a new approach of substrate nanoengineering to produce well-controlled defects. $\mathrm{LaAlO}_{3}$ substrates have been ion-etched with different $34 \mathrm{~nm}$ deep patterns prior to the deposition of the films. An annealing step at $1000^{\circ} \mathrm{C}$ after substrate etching has been undertaken to negate the Ar-ion damage to the surface. The $J_{c}$ of the so-prepared samples measured at different temperatures has been compared to thin films deposited on plain substrates at the same time. In general, an increase in $J_{c}$ is observed across all temperatures and fields as a result of both patterning and annealing. In particular, at zero field and $85 \mathrm{~K}$, the largest enhancement of $>40 \%$ has been recorded for circle and triangle patterns. This new substrate nanoengineering technique is very promising for flux trapping of superconducting devices, particularly because an increase to critical current $\left(I_{c}\right)$ also occurs. Further optimization of depth, size, and shape of the patterns is expected to produce further improvements to $J_{c}$. Published by AIP Publishing.

https://doi.org/10.1063/1.5063261
\end{abstract}

\section{INTRODUCTION}

An important aspect for the functionality of many different superconducting devices is the enhancement of the critical current density $\left(J_{c}\right)$. It allows more efficient current transport and points out an enhanced magnetic flux pinning potential. Extra energy is then required, usually in the form of a Lorentz force $F_{L}$, to depin vortices from these pinning sites. One simple way to artificially create pinning sites is to remove the superconductor in specific, controlled locations creating the so-called antidots (ADs). The primary use of ADs may be in superconducting quantum interference devices (SQUIDs) to reduce the noise associated with vortex movement. ${ }^{1,2}$ Similarly, ADs can be used to reduce energy losses from vortex movement in thin film microwave resonators. ${ }^{3}$ For the optimal vortex pinning potential, the size of the AD should be between double the coherence length $(\xi)$, the size of the vortex, ${ }^{4-6}$ and the penetration depth $(\lambda){ }^{7,8}$ For $\mathrm{YBa}_{2} \mathrm{Cu}_{3} \mathrm{O}_{7}$ (YBCO), this corresponds to the range from $\sim 5 \mathrm{~nm}^{9}$ to $\sim 150 \mathrm{~nm}^{5,10}$ To create ADs of these dimensions, the use of electron beam lithography (EBL) is required. Recently, improvements to $J_{c}$ have also been found using "large" $(2 \mu \mathrm{m}-3 \mu \mathrm{m})$ ADs. ${ }^{1-14}$ In this case, optical lithography may be used, which is technologically a much faster and cheaper process than EBL. The main concern with large ADs is that patterned films occasionally led to degradation. ${ }^{11,12}$ Indeed, this process can be potentially damaging to

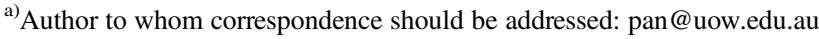

the YBCO from ion implantation, handling, and water exposure. Moreover, even if $J_{c}$ is increased relative to plain (nonpatterned) films, the overall critical current $\left(I_{c}\right)$ may still be reduced due to the removal of a relatively large volume of the superconductor.

To achieve consistent and well-controlled $J_{c}$ enhancement, we propose that substrates can be patterned prior to the deposition of the films. If the superconducting properties inside the etched region of the substrate are similar to those outside, the step between them will act as the additional pinning region. Such substrate nanoengineering can adopt any previous patterns and arrays used for ADs. In this case, the volume of the superconductor remains effectively the same as the corresponding plain film. An increase in $J_{c}$ would also then imply an increase in $I_{c}$. Furthermore, YBCO films would not be treated by a potentially harmful ion/chemical etching process (overheating, loss of oxygen, Ar-ion implantation) and additionally exposed to moisture causing degradation ${ }^{15}$ during handling.

A similar technology is employed to create step-edge Josephson Junctions (SEJs) in YBCO. ${ }^{16}$ This process involves etching a step or a ramp into a substrate to produce SEJs caused by a nanoscale thick, artificially created weak-link. ${ }^{16-18}$ This weak-link separates the top and the ramp part of the step. To form an SEJ, the film thickness to step height ratio $d / h$ must be $\leq 1 .{ }^{19}$ Above this value, a weak-link would not form; hence, no Josephson Junction properties could be expected. In the case of this work, we aim to enhance $J_{c}$ implying no weak-links; hence, we require $d / h \geq 1$. 


\section{EXPERIMENTAL DETAILS}

The high quality YBCO thin films used in this work have been grown by pulsed laser deposition (PLD). The details of our well-established PLD process have been described elsewhere. ${ }^{20-22}$ In this work, $200 \mathrm{~nm}$ thick YBCO films were deposited on $5 \times 5 \mathrm{~mm}^{2} \mathrm{LaAlO}_{3}$ (LAO) substrates, possessing the critical temperature $T_{c}=(90.5 \pm 0.5)$ $\mathrm{K}$ and $J_{c} \simeq 3.5 \times 10^{10} \mathrm{Am}^{-2}$ at $77 \mathrm{~K}$. Optical laser lithography and ion-beam etching were employed to pattern the substrates. These were first covered uniformly by spin coating with a positive photoresist. A pattern was then defined by a UV laser in a Heidelberg Instruments $\mu$ pg 101 micro pattern generator and then developed. After placing the so-prepared substrates in a vacuum chamber and bombarding them with $\mathrm{Ar}^{+}$ions accelerated with an RFM30 ion gun for 10 min, we obtain designed patterns. Taking an atomic force microscopy (AFM) cross section profile gives the depth of an etched hole on average to be $(34.5 \pm 0.5) \mathrm{nm}$.

To negate the damage caused by etching, we have annealed the substrates in a similar fashion to that described by Kawanowa. ${ }^{23}$ The substrates were annealed at $1000{ }^{\circ} \mathrm{C}$ in air for $>2 \mathrm{~h}$ and then were moved immediately to the PLD chamber and a YBCO film was deposited. This type of heat treatment has already been shown to flatten the substrate surface and also resulted in enhancing the quality of the grown YBCO, in particular for ultrathin films. ${ }^{24,25}$

An AFM cross section profile across the hole covered by a deposited film revealed the depth of the hole to remain about $(35.3 \pm 1.8) \mathrm{nm}$. The thickness to step ratio is therefore $200 / 35 \approx 5.7>1$, so a SEJ should not form. ${ }^{19}$

In addition, to ensure a proper characterization, a plain substrate and an annealed plain substrate have also been deposited at the same time for comparison.

In this work, we selected four representative patterns, which were also explored in our previous work, ${ }^{11,12}$ for the proof of the concept purpose, rather than to optimize the expected $J_{c}$ enhancement. These four patterns were etched into different substrates as follows (Fig. 1): (a) squares, (b) graded channels, (c) triangles, and (d) circles, and these were deposited in two batches. The first batch used the substrates patterned with the squares (S1) and graded channels (G1) along with a plain substrate (P1) and an annealed plain substrate (AP1). The second batch used the substrates patterned with triangles (T2) and circles (C2) along with a plain substrate (P2) and an annealed plain substrate (AP2). Having $\mathrm{P} 1,2$ and AP1,2 ensures a proper comparison to the effect of the substrate patterning and its annealing on $T_{c}$ and $J_{c}$, rather than unlikely random errors during depositions. Note that all patterned substrates were annealed as mentioned above.

S1 [Fig. 1(a)] has $10 \mu \mathrm{m}$ sides in a triangular array with a period of $20 \mu \mathrm{m}$ so that there was a $10 \mu \mathrm{m}$ vertical and horizontal gap between squares.

G1 [Fig. 1(b)] is a set of $5 \mu \mathrm{m}$ wide concentric square rings. This was arranged in a way so that the rings were dense in the center of the sample and gradually became sparser toward the edge. The array period starts at $7 \mu \mathrm{m}$ and increased at $1.6 \times$ previous array period so that the period between the final two channels was $825 \mu \mathrm{m}$. A similar
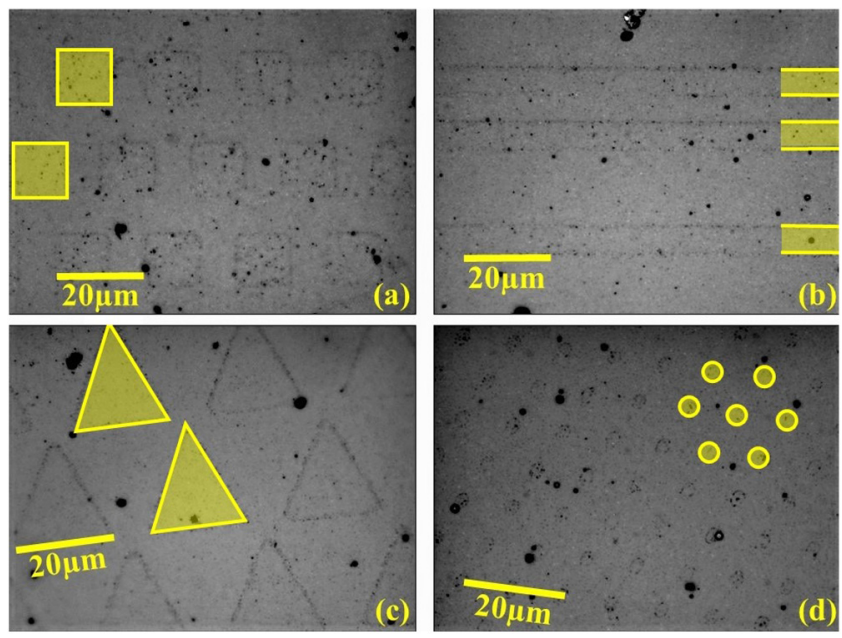

FIG. 1. Optical images of surfaces for all samples with the substrates patterned with (a) squares, (b) graded channels, (c) triangles, and (d) circles. Shaded (yellow) regions emphasize the shape of the etched regions for each pattern, while the edge between the etched and non-etched regions (where the additional pinning is assumed to occur) is shown by a thin solid line and is further explored in Figs. 2(b) and 3.

graded type of ring patterns with ADs of different dimensions has previously been successfully employed for pinning enhancement in different films. ${ }^{11,26}$

T2 [Fig. 1(c)] is the pattern with the largest etched region used having a $20 \mu \mathrm{m}$ base and height. The triangles were also arranged in a triangular array with a horizontal period of $30 \mu \mathrm{m}$ and a vertical period of $25 \mu \mathrm{m}$, which meant a gap of $10 \mu \mathrm{m}$ and $5 \mu \mathrm{m}$ between shapes horizontally and vertically, respectively. It should also be noted that the triangles in $\mathrm{T} 2$ are orientated such that the apex is pointed toward the center of the sample. This orientation is similar to the work done with $\mathrm{ADs}$ in an attempt to produce a ratchet effect, which allows flux to experience a stronger pinning effect entering (increasing field) than exiting (decreasing field). ${ }^{11,12}$

C2 [Fig. 1(d)] has the smallest features created having a $3 \mu \mathrm{m}$ diameter and a period of $10 \mu \mathrm{m}$ in a triangular array.

Measurements of $T_{c}$ and $J_{c}$ were performed in a Quantum Design Physical Properties Measurement System (PPMS). The $T_{c}$ was measured by DC magnetization measurements using an applied magnetic field $B_{a}=2.5 \mathrm{mT}$. The field dependent magnetic moment was measured at temperatures of $10 \mathrm{~K}$, $45 \mathrm{~K}, 77 \mathrm{~K}$, and $85 \mathrm{~K}$. From these measurements, the $J_{c}$ can
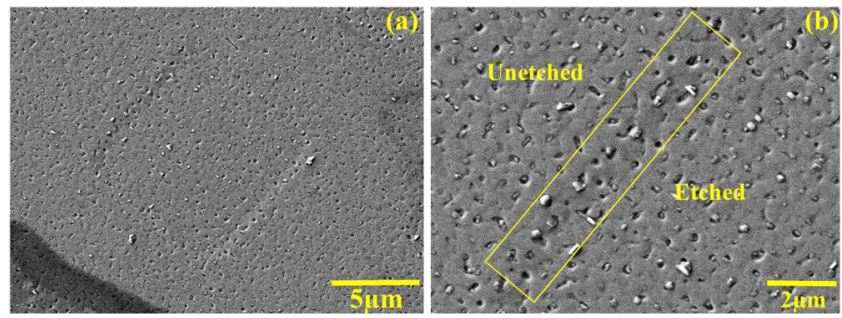

FIG. 2. SEM images of YBCO surfaces for S1 at different magnifications. One full etched square with its surrounds is shown in (a), while (b) displays only one edge of the square. The yellow box in (b) denotes the edge area separating the film deposited on the unetched surface of the substrate and the etched surface of the substrate within the square. 
TABLE I. Comparison of $T_{c}$ for all samples. Also shown is the concentration of surface defects for 4 samples which were imaged using SEM.

\begin{tabular}{lccc}
\hline \hline & $T_{c}$ & Outgrowths $\left(\mu \mathrm{m}^{-2}\right)$ & Pores $\left(\mu \mathrm{m}^{-2}\right)$ \\
\hline P1 & 90 & 1.60 & 2.48 \\
AP1 & 90 & 2.72 & 0.20 \\
S1 & 90.6 & 2.00 & 0.56 \\
G1 & 90.6 & 2.60 & 0.20 \\
P2 & 90.5 & & \\
AP2 & 90.3 & & \\
T2 & 91 & & \\
C2 & 91 & & \\
\hline \hline
\end{tabular}

be calculated using a critical state model for the specific sample geometry used from the equation $J_{c}=3 \Delta M / w$, where $\Delta M$ is the change in magnetization dependent on the field sweep direction and $w$ is the width of one side of the sample. ${ }^{20}$ For all measurements, the field was applied perpendicular to the film plane. Typical $J_{c}$ curves are similar to those shown in Ref. 11.

The scanning electron microscopy (SEM) images were obtained from a dual beam microscopy (Zeiss Aurgia 60). The samples were attached on a conductive holder using a carbon double-sided tape to ensure a proper conducting path and to minimize the charging effect. The images were taken at a medium electron beam voltage of $5 \mathrm{kV}$ to compromise between the image resolution and sample surface contamination.

Cross-sectional Transmission Electron Microscope (TEM) samples were prepared using the in situ lift-out focused ion beam technique and were studied with the help of the FEI Tecnai F20 field emission TEM operated at $200 \mathrm{keV}$.

\section{RESULTS AND DISCUSSION}

Table I somewhat surprisingly shows that the patterned substrate samples have consistently higher $T_{c}$. The overall high $T_{c}$ indicates the high quality of YBCO thin films deposited.

Figure 2 displays the SEM images for S1; however, these are typical for all samples investigated. The SEM images show the large amount of quite common defects on the surface of the YBCO films. These are mostly so-called pin-holes (or pores) and some outgrowths (or droplets). A large number of pin-holes (pores) may be caused by high energy plume adatoms arriving to the substrate due to a short target-to-substrate distance. ${ }^{27}$ This causes short reaction times and inhomogeneous growth with a more pronounced $3 \mathrm{D}$ growth component ${ }^{27,28}$ in the otherwise $2 \mathrm{D} / 3 \mathrm{D}$ Stranski-Krastanov growth mode. ${ }^{29}$ The pores may also be caused by $\mathrm{BaY}_{2} \mathrm{O}_{4}$ secondary phase occlusions. ${ }^{30}$ Outgrowths may be caused by high energies of adatoms in the laser plume, ${ }^{27} \mathrm{CuO}$ and/or $\mathrm{YCuO}_{2}$ phases,${ }^{31}$ or by a stoichiometry mismatch. ${ }^{32}$

The concentration of these defects on the surface was counted within a $5 \times 5 \mu \mathrm{m}^{2}$ area and displayed in Table I. Clearly, the annealing of the substrates has decreased the amount of pores present but increased the outgrowths. Note,

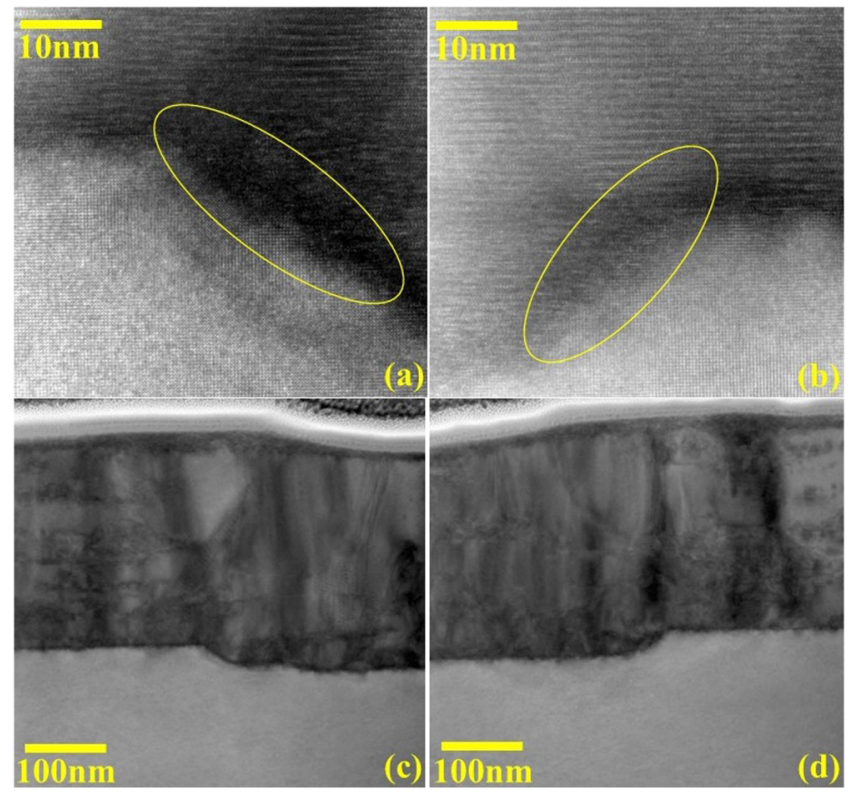

FIG. 3. The TEM cross section of $\mathrm{S} 1$ around the ramp-like edge between etched and unetched regions is shown in high (a) and (b) as well as in lower (c) and (d) magnifications. The direct comparison between the etched and unetched regions indicates that the growth mechanism is similar in both the regions.

only P1 and P2 in Table I have not been annealed. A potential reason for these changes, which will be discussed elsewhere, ${ }^{33}$ stems from the modified surface morphology of the LAO substrate.

It is important to ensure that the etched and unetched regions produce the same high quality YBCO superconducting films. A higher magnification image of the square patterned sample, shown in Fig. 2(b), exhibits the deposited film on both etched and unetched regions of the substrate. The surface of the two regions appears to be very similar. The numbers of defects in the etched area are $1.80 \mu \mathrm{m}^{-2}$ and $0.88 \mu \mathrm{m}^{-2}$ for outgrowths and pin-holes, respectively. Comparison to the non-etched values of $2.00 \mu \mathrm{m}^{-2}$ and $0.56 \mu \mathrm{m}^{-2}$ suggests similar quality structures of the two regions. This is also confirmed by transmission electron microscopy showing very similar columnar growth mode of the film in both the regions [Figs. 3(c) and 3(d)].

The yellow box in Fig. 2(b) shows the ramp-like edge area between the etched and unetched regions, which is where the expected additional pinning potential is. On this edge, the concentration of outgrowths has increased to $2.67 \mu \mathrm{m}^{-2}$ and pin-holes to $2.22 \mu \mathrm{m}^{-2}$.

Figures 3(a) and 3(b) show typical regions near the top of both edges of one of the squares for S1. On the etched side of the substrate, amorphous regions are circled. They do not exhibit a typical layered pattern for the YBCO material, which can be observed above these regions. These amorphous regions are about $5-10 \mathrm{~nm}$ wide, extending along the entire edge of any patterned feature for all samples. They are large enough to accommodate a vortex.

Figures 3(c) and 3(d) display a lower magnification of the same region around the patterned edge. A structural domain wall, produced by the PLD columnar growth of 
YBCO thin films, is clearly seen originating on the tip of the edge traversing up to the surface of the film with a corresponding characteristic feature on the surface of the film, which is likely a pin-hole. The domain walls are also well known to serve as pinning sites for vortices. ${ }^{4,6,34-36}$

For easier clarification of enhancement to $J_{c}$, the difference in the $J_{c}$ is taken between the thin films deposited on their corresponding patterned substrates and $\mathrm{P} 1$ or $\mathrm{P} 2$, shown in Figs. 4(a)-4(d). It is worthwhile reminding that the patterned samples had their substrates annealed before the deposition. Note, a positive difference in Fig. 4 shows enhancement to $J_{c}$, while negative shows degradation. Strikingly, over a broad range of temperature and applied magnetic field, $J_{c}\left(B_{a}\right)$ shows a general enhancement for the films deposited on the patterned substrates with one exception for $\mathrm{T} 2$.

In particular, a higher $J_{c}$ always occurs for patterned samples at very low fields. Most notable enhancements of $\sim 35 \%$ and $\sim 40 \%$ are, respectively, observed for S1 and G1 at $T=85 \mathrm{~K}$. This in itself is a very useful property for many superconducting devices operating close to $T_{c}$. This enhancement is gradually reduced down to under $20 \%$ as the temperature is decreased to $10 \mathrm{~K}$.

Another interesting result is that for $\mathrm{C} 2$, the enhancement degrades at a notably slower rate as the function of the field than for the other patterns at $T<45 \mathrm{~K}$. However, this

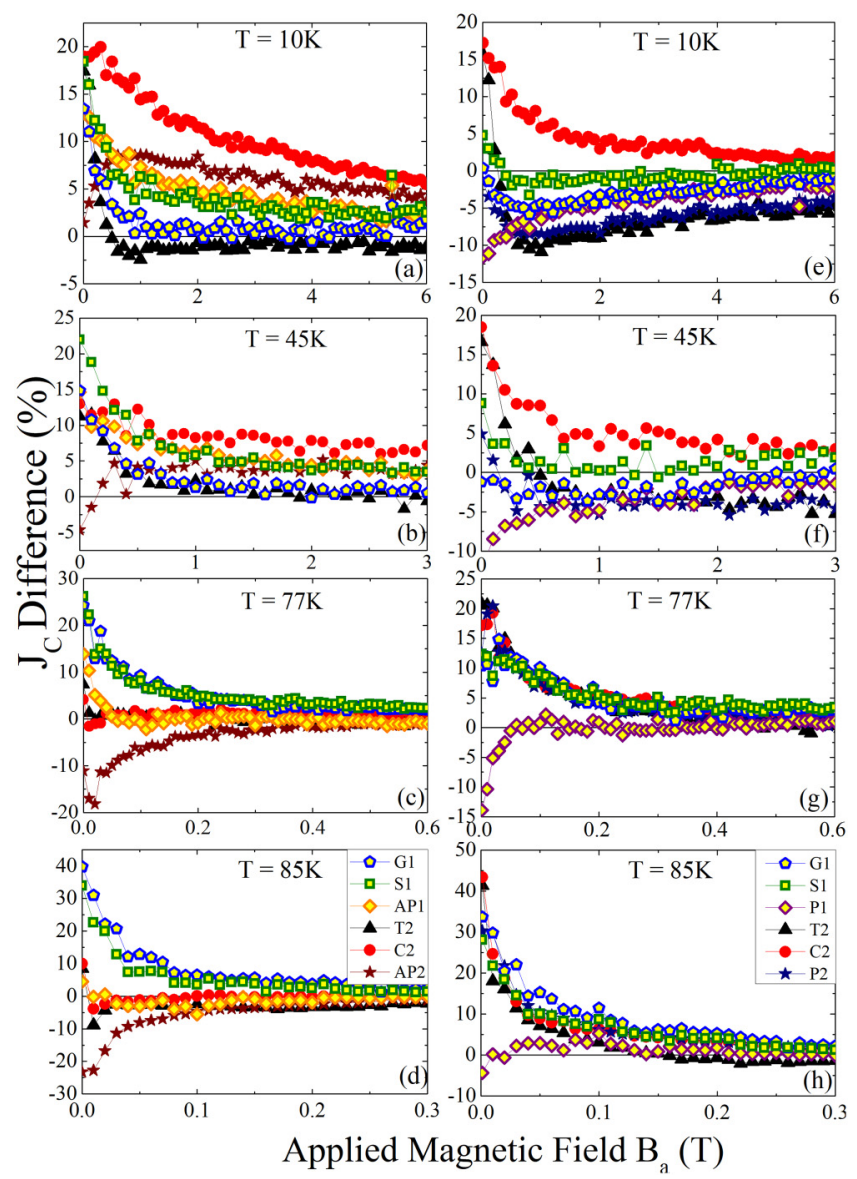

FIG. 4. $J_{c}$ difference of samples compared to the respective plain film P [left column (a)-(d)] and annealed plain film AP [right column (e)-(h)]. G1 denotes graded channels, $\mathrm{S} 1$ squares, $\mathrm{T} 2$ triangles, and $\mathrm{C} 2$ circles. enhancement, which is much larger than for all the other samples at $10 \mathrm{~K}$, significantly deteriorates as the temperature is increased, on the contrary to S1 and G1. To some extent, this behavior is supported by magneto-optical imaging in MoGe films with ADs of a circular shape on one half of the sample and square shape on the other half, ${ }^{37}$ where $J_{c}$ for the part with the circles was found to be higher. These results in the MoGe film are ambiguous since no temperature dependence of this effect was observed due to the flux-jump effect at lower temperatures. It is also notable that the $J_{c}$ difference for the corresponding AP1 and AP2 samples behaves somewhat similarly to $\mathrm{C} 2$ with the only difference that the degradation produces a clearly negative $J_{c}$ difference for $T \geq 77$ $\mathrm{K}$. Such similarity suggests that the pinning mechanism for $\mathrm{C} 2$ and AP1,2 samples is similar. We come back to discussing this point below.

T2 exhibits the exception, showing negative $J_{c}$ difference in $B_{a}>0.5 \mathrm{~T}$ and $T<45 \mathrm{~K}$, which tends to level for higher temperatures, while robustly exhibiting $J_{c}$ enhancement at lower fields over the entire temperature range. The sharp drop in $J_{c}$ with field is a typical result for a film with easy vortex flow channels. ${ }^{38,39}$ The base of the triangles in T2 is orientated to be parallel with the sample edge, while their sides form aligned channels of suppressed superconductivity. These channels enable sliding of vortices toward the triangle vertex and then from one triangle to another toward the center of the sample [Fig. 1(c)]. Vortices breaking away from one triangle corner to a neighboring triangle may be promoted by excessive flux accumulation in the corners of triangles. ${ }^{40}$ This is in stark contrast to the other samples, exhibiting no apparent easy vortex channels.

Furthermore, it is instructive to plot the difference in $J_{c}$ between the same films and their corresponding annealed plain substrates, i.e., either AP1 or AP2 [Figs. 4(e)-4(h)]. Since both the films and plain substrates were deposited on annealed substrates, the remaining difference is only due to the effect of substrate nanoengineering, whereas Figs. 4(a)-4(d) show the superposition of the pinning changes due to the patterning and due to changes associated with annealing.

There are two main features to distinguish in the $J_{c}$ difference plots in Figs. 4(e)-4(h) as follows. (i) The trend of the $J_{c}$ difference as a function of the field is similar for all the samples and (ii) except for $\mathrm{C} 2$. This is best seen at $T=10 \mathrm{~K}$, for which C2 is the only sample showing positive $J_{c}$ difference in the entire field range. As the temperature is increased, all the patterned samples gain positive $J_{c}$ difference, so that the strongest $J_{c}$ enhancement (up to $45 \%$ ) is observed at a highest measured temperature of $85 \mathrm{~K}$. Notably, the behavior of the $J_{c}$ difference becomes very similar for $85 \mathrm{~K}$, including $\mathrm{C} 2$.

Thus, C2 stands out in Fig. 4, indicating that the added pinning contribution is most effective at lowest temperatures for this sample. This type of $J_{c}$ enhancement is most likely associated only with vortex pinning arising at the etching edges, rather than with collective effects of vortex pinning and dynamics facilitated by particularities of the entire pattern as, for example, described for $\mathrm{T} 2$ above and in Refs. 11, 26, 38, and 39. In Ref. 11, for example, nonhomogeneous, non-Bean-like magnetic flux penetration with 
"vortex vacuum" regions appeared as a result of AD pattern contributions to vortex pinning has been proposed. In the case of the circle pattern in $\mathrm{C} 2$, it has the most symmetric shape of its features, the smallest dimensions, the most homogeneous array pattern, and the least area of etching, compared to the other samples investigated in this work. Hence, this pattern is the least suitable for similar collective effects.

T2 shows the weakest performance enhancement and even negative $J_{c}$ difference, especially at lowest measured temperatures and high fields. The positive $J_{c}$ difference is only achieved at low fields $(<0.4 \mathrm{~T})$. However, this pattern still shows the general trend of the $J_{c}$ performance as for the other patterns $(\mathrm{G} 1, \mathrm{~S} 1)$. In fact, even $\mathrm{C} 2$ tends to exhibit the same trend as all other samples in Fig. 4 at $T>45 \mathrm{~K}$.

The empirical analysis of the obtained results indicates that the $J_{c}$ enhancement has a similar origin in all the samples associated with the influence of the patterning. These added factors associated with patterns are likely a synergy of (i) additional vortex pinning sites (or regions) at the edges of the nanoengineered patterns and (ii) the nonhomogeneous vortex penetration and modified non-Bean-like critical state pinning model of vortices in the samples. ${ }^{11}$ The non-homogeneous vortex penetration was shown to be equally effective at any temperature below $T_{c}$ for $\mathrm{AD}$ patterns in Ref. 11. In the present work, the superconducting films do not lose integrity within the patterns as was the case for ADs. Hence, at low temperatures, this non-homogeneous vortex penetration dynamics may not be effective, and, mainly, the added pinning sites by the pattern edges contribute to $J_{c}$ enhancement. At higher temperatures, the vortex dynamics has a more profound influence; hence, the $J_{c}$ enhancement is larger due to the collective influence of the patterns on vortex pinning. This scenario is further supported by $\mathrm{C} 2$, which as mentioned above should be least susceptible to inhomogeneous penetration due to its most "homogeneous" pattern: the circular sample of features, their smallest dimensions, and the least etched area (per feature). Indeed, C2 demonstrates behavior which is least consistent with the non-Bean-like pinning model, and, on the other hand, it is more consistent with the added pinning sites of a certain type, which enhances $J_{c}$ at low temperatures only.

\section{CONCLUSION}

In conclusion, we propose a new, well-controlled substrate nanoengineering approach to enhance $J_{c}$ in superconducting YBCO thin films. Different patterns (circles, squares, triangles, and graded concentric square rings) etched on substrates demonstrate spectacular $J_{c}$ enhancements of up to $45 \%$ in the vicinity of $T_{c}$. The enhancement is the result of introducing additional defects at the pattern edges and collective pinning pattern influence on vortex dynamics. Since YBCO films do not lose their integrity because the pattern manufacturing takes place prior to film deposition, the $J_{c}$ enhancement corresponds to the engineering $I_{c}$, which is an important result for any potential device applications. The growth of the YBCO inside the etched region appears to be the same as the growth outside. Optimization of depth, shape, and dimensions of the patterns is expected to enable further $J_{c}$ control and enhancements.

\section{ACKNOWLEDGMENTS}

This work is supported by the Faculty of Engineering and Information Sciences and Australian Institute for Innovative Materials (University of Wollongong, Australia) and the Australian Government Research Training Program Award. A. Jones has benefited from CSIRO postgraduate top-up scholarship. The authors would like to thank I. Rudnev, W. A. Ortiz, and V. V. Moshchalkov for stimulating discussions, as well as eCOST Action MP1201 on Nanoscale Superconductivity for a supportive research environment.

${ }^{1} \mathrm{P}$. Selders and R. Wördenweber, "Low-frequency noise reduction in $\mathrm{YBa}_{2} \mathrm{Cu}_{3} \mathrm{O}_{7-\delta}$ superconducting quantum interference devices by antidots," Appl. Phys. Lett. 76, 3277-3279 (2000).

${ }^{2} \mathrm{R}$. Wördenweber and P. Selders, "Reduction of low-frequency noise in high- $T_{c}$ SQUIDs by artificial defects," Physica C 366, 135-146 (2002).

${ }^{3}$ D. Bothner, C. Clauss, E. Koroknay, M. Kemmler, T. Gaber, M. Jetter, M. Scheffler, P. Michler, M. Dressel, D. Koelle, and R. Kleiner, "Reducing vortex losses in superconducting microwave resonators with microsphere patterned antidot arrays," Appl. Phys. Lett. 100, 012601 (2012).

${ }^{4}$ V. M. Pan and A. V. Pan, "Vortex matter in superconductors," Fiz. Niz. Temp. 27, 991 (2001) [Low Temp. Phys. 27, 732 (2001) (in Russian)].

${ }^{5}$ A. V. Pan et al., "Thermally activated depinning of individual vortices in $\mathrm{YBa}_{2} \mathrm{Cu}_{3} \mathrm{O}_{7}$ superconducting films," Physica C 407, 1016 (2004).

${ }^{6}$ G. Blatter, M. V. Feigel'man, V. B. Geshkenbein, A. I. Larkin, and V. M. Vinokur, "Vortices in high-temperature superconductors," Rev. Mod. Phys. 66, 1125 (1994).

${ }^{7}$ N. Takezawa and K. Fukushima, "Optimal size of an insulating inclusion acting as a pinning center for magnetic flux in superconductors: Calculation of pinning force," Physica C 290, 31-37 (1997).

${ }^{8}$ V. V. Moshchalkov, M. Baert, V. V. Metlushko, E. Rosseel, M. J. Van Bael, K. Temst, Y. Bruynseraede, and R. Jonckheere, "Pinning by an antidot lattice: The problem of the optimum antidot size," Phys. Rev. B 57, 3615-3622 (1998).

${ }^{9}$ H. Jiang, T. Yuan, H. How, A. Widom, C. Vittoria, and A. Drehman, "Measurements of anisotropic characteristic lengths in YBCO films at microwave frequencies," J. Appl. Phys. 73, 5865-5867 (1993).

${ }^{10}$ R. Prozorov, R. W. Giannetta, A. Carrington, P. Fournier, R. L. Greene, P. Guptasarma, D. G. Hinks, and A. R. Banks, "Measurements of the absolute value of the penetration depth in high- $\mathrm{T}_{c}$ superconductors using a

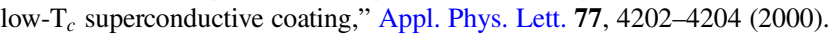

${ }^{11}$ J. George, A. Jones, M. AlQurainy, S. A. Fedoseev, A. Rosenfeld, and A. V. Pan, "Tunable pinning effects produced by nonuniform antidot arrays in YBCO thin films," Ann. Phys. 529, 1600283 (2017).

${ }^{12}$ A. Jones, J. George, S. A. Fedoseev, S. K. H. Lam, J. Du, and A. V. Pan, "Changing the critical current density and magnetic properties of $\mathrm{YBa}_{2} \mathrm{Cu}_{3} \mathrm{O}_{7}$ by using large antidots," IEEE Trans. Appl. Supercond. 28, 2808439 (2018).

${ }^{13}$ A. N. Moroz, A. N. Maksimova, V. A. Kashurnikov, and I. A. Rudnev, "Effects of $\mathrm{Zr}$ doping on magnetic and structural properties of $\mathrm{DyBa}_{2} \mathrm{Cu}_{3} \mathrm{O}_{7-\delta}$ thin films," IEEE Trans. Appl. Supercond. 28, 8000705 (2018).

${ }^{14}$ A. Palau, C. Monton, V. Rouco, X. Obradors, and T. Puig, "Guided vortex motion in $\mathrm{YBa}_{2} \mathrm{Cu}_{3} \mathrm{O}_{7}$ thin films with collective ratchet pinning potentials," Phys. Rev. B 85, 012502 (2012).

${ }^{15}$ D. R. Lundy, L. J. Swartzendruber, and L. H. Bennett, "A brief review of recent superconductivity research at NIST," J. Res. Natl. Inst. Stand. Technol. 94, 147 (1989).

${ }^{16}$ A. V. Pan, O. V. Shcherbakova, S. A. Fedoseev, I. A. Golovchanskiy, D. Attard, S. K. H. Lam, J. Du, C. P. Foley, S. Rubanov, and A. Suvorova, "Enhancing properties of high temperature superconducting step edge Josephson junctions by nanomultilayers with a small mismatch," Adv. Mater. Interfaces 1, 1300112 (2014). 
${ }^{17}$ K. Herrmann, Y. Zhang, H.-M. Muck, J. Schubert, W. Zander, and A. I. Braginski, "Characterization of $\mathrm{YBa}_{2} \mathrm{Cu}_{3} \mathrm{O}_{7}$ step-edge Josephson junctions," Supercond. Sci. Technol. 4, 583 (1991).

${ }^{18}$ C. P. Foley, E. E. Mitchell, S. K. H. Lam, B. Sankrithyan, Y. M. Wilson, D. L. Tilbrook, and S. J. Morris, "Fabrication and characterisation of YBCO single grain boundary step edge junctions," IEEE Trans. Appl. Supercond. 9, 4281 (1999).

${ }^{19}$ E. E. Mitchell and C. P. Foley, "YBCO step-edge junctions with high $I_{c} R_{n}$, , Supercond. Sci. Technol. 23, 065007 (2010).

${ }^{20}$ A. V. Pan, S. Pysarenko, and S. X. Dou, "Drastic improvement of surface structure and current-carrying ability in $\mathrm{YBa}_{2} \mathrm{Cu}_{3} \mathrm{O}_{7}$ films by introducing multilayered structure," Appl. Phys. Lett. 88, 232506 (2006).

${ }^{21}$ A. V. Pan, S. V. Pysarenko, D. Wexler, S. Rubanov, and S. X. Dou, "Multilayering and Ag-doping for properties and performance enhancement in $\mathrm{YBa}_{2} \mathrm{Cu}_{3} \mathrm{O}_{7}$ films," IEEE Trans. Appl. Supercond. 17, 3583 (2007).

${ }^{22}$ I. A. Golovchanskiy, A. V. Pan, S. A. Fedoseev, and M. Higgins, "Significant tunability of thin film functionalities enabled by manipulating magnetic and structural nano-domains," Appl. Surf. Sci. 311, 549 (2014).

${ }^{23}$ H. Kawanowa, H. Ozawa, M. Ohtsuki, Y. Gotoh, and R. Souda, "Structure analysis of $\mathrm{LaAlO}_{3}(001)$ surfaces by low energy neutral scattering spectroscopy," Surf. Sci. 506, 87 (2002).

${ }^{24} \mathrm{X}$. X. Xi, J. Geerk, G. Linker, Q. Li, and O. Meyer, "Preparation and superconducting properties of ultrathin $\mathrm{YBa}_{2} \mathrm{Cu}_{3} \mathrm{O}_{7-x}$ films," Appl. Phys. Lett. 54, 2367 (1989).

${ }^{25}$ B. H. Moeckly, S. E. Russek, D. K. Lathrop, R. A. Buhrman, M. G. Norton, and C. B. Carter, "Growth and properties of $\mathrm{YBa}_{2} \mathrm{Cu}_{3} \mathrm{O}_{7}$ thin films on vicinal and polycrystalline $\mathrm{MgO}$ substrates," Appl. Phys. Lett. 57, 2951 (1990)

${ }^{26}$ M. Motta, F. Colauto, W. A. Ortiz, J. Fritzsche, J. Cuppens, W. Gillijns, V. V. Moshchalkov, T. H. Johansen, A. Sanchez, and A. V. Silhanek, "Enhanced pinning in superconducting thin films with graded pinning landscapes," Appl. Phys. Lett. 102, 212601 (2013).

${ }^{27}$ S. V. Pysarenko, A. V. Pan, and S. X. Dou, "Origin of surface morphology variation during pulsed laser deposition of $\mathrm{YBa}_{2} \mathrm{Cu}_{3} \mathrm{O}_{7}$ superconducting films," IEEE Trans. Appl. Supercond. 21, 3179 (2011).

${ }^{28}$ M. Hawley, I. D. Raistrick, J. G. Beery, and R. J. Houlton, "Growth mechanism of sputtered films of YBCO studied by scanning tunneling microscopy," Science 251, 1587 (1991).

${ }^{29}$ I. N. Stranski and L. Von Krastanov, "Abhandlungen der MathematischNaturwissenschaftlichen Klasse,” Akad. Wiss. Lit. Mainz 146, 797 (1939).
${ }^{30}$ K. Develos-Bagarinao, H. Yamasaki, J. C. Nie, and Y. Nakagawa, "Thickness dependence of $\mathrm{J}_{c}$ for $\mathrm{YBCO}$ thin films prepared by large-area pulsed laser deposition on $\mathrm{CeO}_{2}$-buffered sapphire substrates," Supercond. Sci. Technol. 18, 667 (2005).

${ }^{31}$ K. D. Develos, H. Yamasaki, A. Sawa, and Y. Nakagawa, "On the origin of surface outgrowths in pulsed-laser-deposited $\mathrm{YBCO} / \mathrm{CeO}_{2} / \mathrm{Al}_{2} \mathrm{O}_{3}$ thin films," Physica C 361, 121 (2001).

${ }^{32}$ H. Xin, D. E. Oates, A. C. Anderson, A. R. L. Slattery, G. Dresselhaus, and M. S. Dresselhaus, "Comparison of power dependence of microwave surface resistance of unpatterned and patterned YBCO thin film," IEEE Trans. Microw. Theory Techn. 48, 1221 (2000).

${ }^{33}$ A. Jones, S. K. H. Lam, J. Du, S. Rubanov, and A. V. Pan, "Modification of pinning in $\mathrm{YBa}_{2} \mathrm{Cu}_{3} \mathrm{O}_{7}$ thin films by substrate annealing," IEEE Trans. Appl. Supercond. (submitted).

${ }^{34}$ V. Pan, Y. Cherpak, V. Komashko, S. Pozigun, C. Tretiatchenko, A. Semenov, E. Pashitskii, and A. V. Pan, "Supercurrent transport in $\mathrm{YBa}_{2} \mathrm{Cu}_{3} \mathrm{O}_{7-\delta}$ epitaxial thin films in a dc magnetic field," Phys. Rev. B 73, 054508 (2006).

${ }^{35}$ A. V. Pan, S. Pysarenko, and S. X. Dou, "Quantitative description of critical current density in YBCO films and multilayers," IEEE Trans. Appl. Supercond. 19, 3391 (2009).

${ }^{36}$ I. A. Golovchanskiy, A. V. Pan, O. V. Shcherbakova, S. A. Fedoseev, and S. X. Dou, "An all-field-range description of the critical current density in superconducting YBCO films," Supercond. Sci. Technol. 24, 105020 (2011).

${ }^{37}$ M. Motta, F. Colauto, J. I. Vestgården, J. Fritzsche, M. Timmermans, J. Cuppens, C. Attanasio, C. Cirillo, V. V. Moshchalkov, J. Van de Vondel, T. H. Johansen, W. A. Ortiz, and A. V. Silhanek, "Controllable morphology of flux avalanches in microstructured superconductors," Phys. Rev. B 89, 134508 (2014)

${ }^{38}$ V. R. Misko and F. Nori, "Magnetic flux pinning in superconductors with hyperbolic-tessellation arrays of pinning sites," Phys. Rev. B 85, 184506 (2012).

${ }^{39}$ Y. L. Wang, M. L. Latimer, Z. L. Xiao, R. Divan, L. E. Ocola, G. W. Crabtree, and W. K. Kwok, "Enhancing the critical current of a superconducting film in a wide range of magnetic fields with a conformal array of nanoscale holes," Phys. Rev. B 87, 220501 (2013).

${ }^{40}$ F. Colauto, J. I. Vestgården, A. M. H. de Andrade, A. A. M. Oliveira, W. A. Ortiz, and T. H. Johansen, "Limiting thermomagnetic avalanches in superconducting films by stop-holes," Appl. Phys. Lett. 103, 032604 (2013). 